\title{
Proximity to Mosquito Breeding Habitat and Ross River Virus Risk in the Peel Region of Western Australia
}

\author{
Andrew Jardine, Peter J. Neville, and Michael D.A. Lindsay²
}

\begin{abstract}
It is intuitive that vector-borne disease exposure risk is related to proximity to sources of vector breeding, but this aspect rarely receives empirical testing. The population of Western Australia (WA) is increasing rapidly, with many new residential developments proposed in close proximity to mosquito breeding habitat. However, potential mosquito-borne disease risks for future residents are given little consideration by planning authorities. The Peel region is one of the fastest growing regions in WA and regularly experiences a large number of cases of the mosquito-borne Ross River virus (RRV) disease with epidemics occuring in the region every few years. A spatial analysis of RRV disease data in the Peel region was undertaken to determine the risk associated with proximity to a mosquito breeding habitat. Geographic Information Systems (GIS) software was used to create buffers between 1 and $6 \mathrm{~km}$ from the breeding habitat. The number of cases per 1000 dwellings in each buffer was calculated between 2002/03 to 2011/12 for years with $>100$ cases across all buffers $(n=5)$ in addition to the cumulative rate over the entire period in each buffer. Residents living within $1 \mathrm{~km}$ of a mosquito breeding habitat had a significantly higher rate of RRV disease compared to the background rate across the Peel region in all individual years investigated. The cumulative data over the 10-year study period showed that residents in the 1- and 2-km buffers had a significantly higher rate, whereas those living between 3 and $6 \mathrm{~km}$ away did not. This study demonstrates an increased mosquito-borne disease risk associated with living in close proximity to a mosquito breeding habitat in a rapidly expanding region of WA and highlights the importance of considering mosquito-borne disease risks when planning authorities assess new residential development applications. Known mosquito breeding wetlands should be incorporated into land use planning scheme maps to ensure that they are accurately delineated and the implications are considered when planning decisions are made.
\end{abstract}

Key Words: Mosquitoes—Geographic Information Systems (GIS)—Epidemiology—Arbovirus.

\section{Introduction}

B URGEONING POPULATION GROWTH and associated urban expansion present significant challenges to local government and planning authorities globally. One often neglected outcome of such urban expansion is heightened disease risk as human populations encroach closer to natural mosquito breeding habitats. For example, an increased risk of malaria in Africa (Staedke et al. 2003, Midega et al. 2012) and Asia (Haque et al. 2009) has been demonstrated with decreasing distance to mosquito breeding habitats; living in a residence located within 100 meters from one or more tree hole breeding sites was demonstrated to be associated with almost four times greater risk of La Crosse encephalitis infection in eastern Ten- nessee (Erwin et al. 2002); and areas in southeast Queensland with a greater proportion of wetlands and native vegetation and levels of adult mosquito activity have been associated with higher rates of Ross River virus (RRV; Togaviridae: Alphavirus) (Muhar et al. 2000, Ryan et al. 2006, Hu et al. 2010). These studies exemplify the risks associated with living in proximity to sources of vector populations. In areas experiencing significant population and urban expansion, there is a critical need for research evaluating such links and for associated evidence and guidance to be provided to planning authorities.

Western Australia (WA) was the fastest growing state in Australia between 2003 and 2013, with a population increase of $29 \%$ compared to $17 \%$ nationally over this period (Australian Bureau of Statistics 2014). Much of this population

\footnotetext{
${ }^{1}$ Mosquito-Borne Disease Control, Environmental Health Hazards Unit, Environmental Health Directorate, Department of Health Western Australia, Western Australia, Australia.

${ }^{2}$ Environmental Health Hazards Unit, Environmental Health Directorate, Department of Health Western Australia, Western Australia, Australia.
} 
growth occurred in the outer suburbs of Perth, most notably in the Peel region (defined here as the Local Government Areas of Rockingham, Mandurah, Murray, and Waroona; Fig. 1), and it increased by 53\% between 2003 and 2013 (Australian Bureau of Statistics 2014). Population forecasts by the Western Australian Planning Commission indicate that this rapid growth rate is expected to continue in the region over coming decades (Western Australian Planning Commission 2012), placing further pressure to develop surrounding land into new residential areas that are often proposed close to water due to intrinsic aesthetic values. Residents who live in close proximity to breeding sites may be subjected to intense nuisance problems and, more importantly, increased risk of infection with a mosquito-borne virus.

A substantial number of cases of RRV (Togaviridae: Alphavirus) disease, a nonfatal but potentially debilitating polyarthritic disease (Russell 2002), are reported annually from the Peel region, and large outbreaks are experienced every few years despite significant mosquito control efforts (Lindsay et al. 1996). Extensive areas of salt marsh mosquito breeding habitat are located in the lower reaches of the Serpentine River, the Peel Inlet, and the Harvey Estuary (Fig. 1) (Wright 1988), many of which are protected under the Ramsar Convention listed Peel-Yalgorup system (Department of the Environment 2011). Mosquito surveillance in the Peel region over the last 20 years has shown that Aedes (Ae.) camptorhynchus is the predominant species between April and December, whereas Ae. vigilax is the major species present from January to March each year (Lindsay et al. 1996). Both species are important vectors of RRV in coastal regions of WA (Lindsay et al. 1996, Russell 2002). Furthermore, substantial areas of native bushland remain in close proximity to many mosquito breeding sites in the Peel region that support western grey kangaroo populations (Macropus fuliginosus), an important vertebrate host of RRV in southern WA (Russell 2002, Potter et al. 2014).

Significant residential development has occurred and continues to expand in close proximity to mosquito breeding habitats in the Peel region due to the aesthetic values of living near water. Unfortunately, the potential for residents who move into these areas to be exposed to mosquito-borne diseases and intense nuisance problems is given little consideration by planning authorities because limited evidence presently exists to quantify this risk. In WA, RRV incidence was found to decrease with distance from a tidal estuary with extensive mosquito breeding habitats in rural and semirural areas (Vally et al. 2012). However, no relationship was found in urban areas with higher population density (Vally et al. 2012). We also recently demonstrated a significant increase in RRV risk within $2 \mathrm{~km}$ of a known highly productive breeding habitat located $200 \mathrm{~km}$ south of the current study region that resulted from dispersal of Ae. camptorhynchus into surrounding residential areas (Jardine et al. 2014).

The aim of the current study is to build on these previous findings and quantify the risk associated with proximity to mosquito breeding habitats in the Peel region, a key focus of RRV activity and population growth. The larger spatial scale and more detailed temporal analysis in this study add to the evidence basis to inform planning decisions and target mosquito control activities.

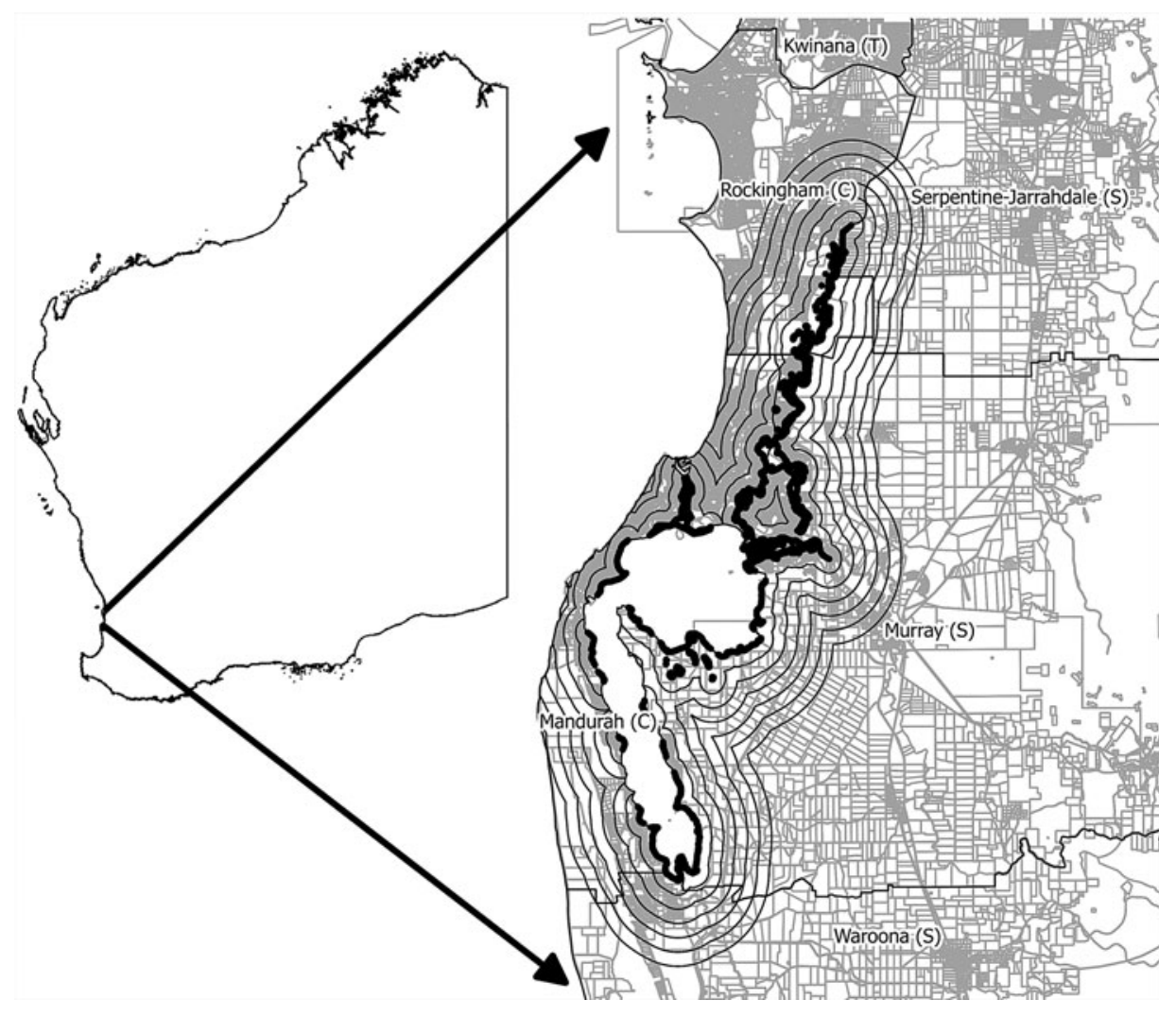

FIG. 1. Location of the study site within the Peel Region with 1- to 6-km buffer zones indicated by the solid black lines surrounding known mosquito breeding wetlands of the Peel and Harvey Estuaries. 


\section{Materials and Methods}

RRV is a notifiable disease in Australia, meaning that all cases of RRV diagnosed by a doctor or detected in laboratory tests in WA are required to be notified to the Department of Health under the Health Act (1911). WA also undertakes an "enhanced surveillance" program through which RRV cases are followed up via patient interviews to determine the most likely location and timing of exposure. Residential address is assumed to be the location of exposure if the case cannot be contacted.

The dataset created for spatial analyses consisted of all cases for which the most likely place of exposure could be precisely geocoded to a specific cadastral lot (a legally defined property boundary). In addition, if place of exposure data or residential data are not given as an exact location but can be pinpointed with reasonable confidence (e.g., a street corner within 250 meters), then these cases are also geocoded. All other cases were excluded from the dataset for spatial analyses.

Mosquito breeding habitats identified from field surveys in the Peel Inlet, Harvey Estuary, and the lower reaches of the Serpentine River (Wright 1988) were mapped, and six 1-km buffers were created around them using Quantum Geographic Information System (GIS) 1.7.4 (Quantum GIS Development Team 2012) (Fig. 1). The intersection of the buffers with the RRV case data was used to determine the number of cases within each buffer.

A property street address cadastral lot data layer maintained by Landgate was overlaid by the Peel Regional Planning Scheme data layer maintained by the Western Australian Planning Commission. Addresses in areas zoned as urban or rural were retained and all other lots were excluded. Any other addresses not defined as a house were also excluded. Finally, the remaining property street address lots were intersected with the buffers to determine the number of dwellings within each buffer.

The case and cadastral data were then summed for each buffer, the rate of RRV notifications per 1000 dwellings was determined, and 95\% confidence intervals (CIs) were calculated. Poisson regression was then undertaken to determine if a statistically significant trend in the rate of RRV notifications with buffer distance was present. The number of cases was assigned as the dependent variable, the buffer distance as the independent variable, and the number of dwellings as the exposure variable.

Finally, to determine the expected background rate for the Peel region, the number of RRV cases and dwellings for the whole region was calculated using the same methods described above. The rate for each buffer was determined to be significantly elevated if the $95 \%$ CI did not span the background rate.

Rates were calculated for cases recorded between July 1, 2002, and June 30, 2012, to establish the cumulative risk for each buffer over the 10-year period. The same analysis was also undertaken for individual financial years in which more than 100 cases were notified across all buffers, which included 2005/ 06, 2007/08, 2008/09, 2010/11, and 2011/12 mosquito seasons, to determine if the pattern repeated in all outbreak years.

Ethics approval was not required because our study evaluated data collected during the routine public health response to RRV as a notifiable disease.

\section{Results}

The background rate of RRV per 1000 dwellings across the Peel region from July, 2002, to June, 2012, was 0.93 cases per
Table 1. Number of Dwellings, Ross River

Virus Cases, and RATE PeR 1000 DWellings by Buffer Distance from Mosquito Breeding Habitat, Peel Region, July, 2002, to June, 2012

\begin{tabular}{lrrr}
\hline & & \multicolumn{2}{c}{ July, 2002, to June, 2012} \\
\cline { 3 - 4 } $\begin{array}{l}\text { Buffer } \\
\text { distance }\end{array}$ & Dwellings & Cases & $\begin{array}{c}\text { Average annual } \\
\text { cases/1000 dwellings }\end{array}$ \\
\hline$<1 \mathrm{~km}$ & 20,128 & 381 & 1.89 \\
$1-2 \mathrm{~km}$ & 17,724 & 209 & 1.18 \\
$2-3 \mathrm{~km}$ & 8,555 & 94 & 1.10 \\
$3-4 \mathrm{~km}$ & 4,605 & 38 & 0.83 \\
$4-5 \mathrm{~km}$ & 6,874 & 45 & 0.65 \\
$5-6 \mathrm{~km}$ & 4,178 & 25 & 0.60 \\
Background & & & 0.93 \\
\hline
\end{tabular}

year, and average annual rates for each buffer are shown in Table 1. A clear decreasing trend with increasing buffer distance was evident (Fig. 2), and Poisson regression indicated this trend to be significant $(p<0.001)$. The number of RRV cases per 1000 dwellings was significantly higher than the rate across the Peel region for buffer distances up to $2 \mathrm{~km}$ during the 10-year period.

Figure 3 shows the number of RRV cases per 1000 dwelling for each buffer distance for the years in which more than 100 cases were notified across all buffers, which included 2005/06, 2007/08, 2008/09, 2010/11, and 2011/12 mosquito seasons. It was consistently demonstrated across all years investigated that residents living within $1 \mathrm{~km}$ of a mosquito breeding habitat had a significantly higher rate of RRV disease compared to the background rate across the Peel region. Poisson regression also indicated that a statistically significant reduction $(p<0.05)$ in $\mathrm{RRV}$ rate with increasing buffer distance was evident in all years except 2011/12.

\section{Discussion}

This study sought to quantify disease risk associated with proximity to sources of vector breeding and a significantly increased risk of contracting RRV associated with living in close proximity to a mosquito breeding salt marsh habitat in the Peel region of WA was demonstrated. Between July, 2002, and June, 2012, the rate of RRV cases per 1000 dwellings within $1 \mathrm{~km}$ of a breeding habitat was double the rate compared to the Peel region as a whole, and $26 \%$ higher between 1 and $2 \mathrm{~km}$ from a mosquito breeding habitat. A significant decreasing trend with increasing buffer distance was evident over the 10 -year period and in most individual years with high case numbers $(>100)$.

Only one year, 2011/12, was divergent from this trend. In this year, a cluster of cases occurred in the 5- to 6-km buffer, which was thought to be associated with a separate breeding site, not related to those in the Peel Inlet or Harvey Estuary that were mapped as part of this study. Given that this year was otherwise comparable with the other high case number years (i.e., case numbers in the 1-km buffer were still significantly higher than the background rate and the other buffers were not different from the background rate), we consider this an atypical event for this region. Overall, the data show that those living within $2 \mathrm{~km}$ of a mosquito breeding habitat were at increased risk of RRV disease, 


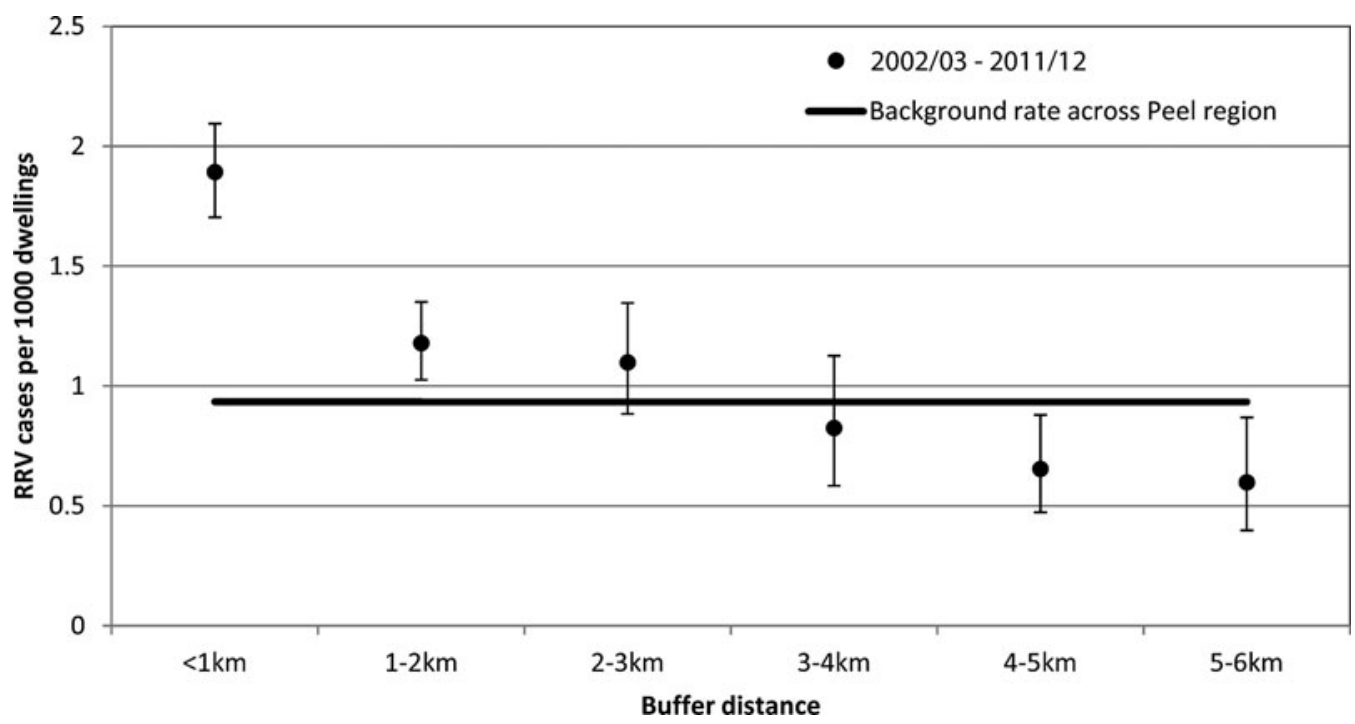

FIG. 2. Number of Ross River virus (RRV) cases per 1000 dwellings versus buffer distance from a mosquito breeding habitat, Peel region, July, 2002, to June, 2012.

which is further evidence to support the results of previous spatial analyses of RRV case data in close proximity to Ae. camptorhynchus breeding habitats in other locations in southwestern WA (Vally et al. 2012, Jardine el al. 2014).

With increasing pressure to release land for residential development in the Peel region, these findings provide evidence to planning authorities for consideration when assessing applications for new residential developments in close proximity of recognized permanent or semipermanent natural mosquito breeding sites, such as wetlands, salt marshes, or estuarine environments. It is recommended that planning authorities incorporate known mosquito breeding wetlands into land use planning scheme maps to ensure that they are accurately delineated and the implications are considered when planning decisions are made. Notifications should be placed on the land titles within $2 \mathrm{~km}$ of known mosquito breeding habitats to advise prospective buyers of the increased health risk. Built form design measures such as insect screening on doors and windows and screened outdoor enclosures, public education packages, and public signage should also be included as part of the conditions of approval. Physical changes to topography should prevent runoff from creating surface retention and constructed water bodies should be located, designed, and maintained so they do not create or contribute to additional mosquito breeding. It may also be appropriate for developers of land in close proximity to breeding habitats to contribute to the cost of mosquito control by the local authority.

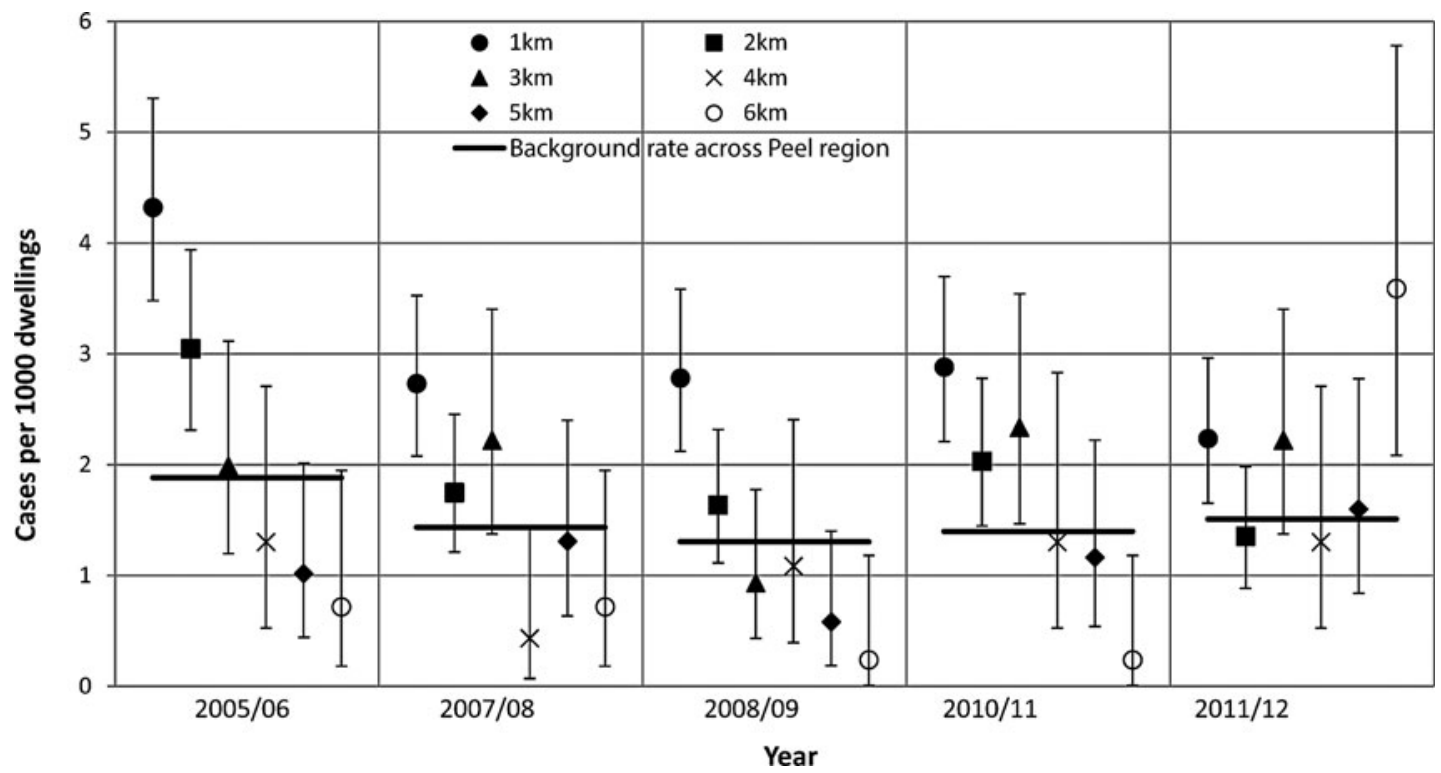

FIG. 3. Number of Ross River virus cases per 1000 dwellings versus buffer distance during years in which more than 100 cases were notified across all buffers. 
It is also recommended that environmental managers of natural wetlands that support mosquito breeding should be made aware that mosquito management actions that may impact on the wetlands could be required if developments in close proximity are approved to proceed. Where possible, public open space with limited vegetation should be located between a mosquito breeding habitat and residential areas to create an area that is refractory to mosquito dispersal, thereby reducing the number of mosquitoes impacting residents. Finally, similar to the proposal for bushfire management plans to be required for planning development in high fire danger areas in WA (Department of Planning 2014), a mosquito management plan should be developed in high-risk mosquito-borne disease areas or for developments in close proximity to mosquito breeding sites to ensure that viable measures have been considered and can be applied to reduce the risk of exposure to disease carrying mosquitoes.

Although we are confident in the robustness of the findings of this study, there are also limitations that should be acknowledged. Limitations include the accuracy of exposure location for the RRV data and the need to use dwelling counts to approximate the population at risk. Follow-up data was not available for about half the cases, therefore residential address was assumed to be the location of exposure. An analysis of RRV case data since 2002 showed that where enhanced surveillance information was available, location of exposure was at the place for residence for $66.2 \%$ of cases (unpublished data). Therefore, whereas the exposure location of some of the RRV cases included in this study will not be accurate, the overall proportion will be relatively small. Furthermore, there is no reason to suspect the proportion of cases with inaccurate exposure information would have varied across the buffers and therefore the potential for differential bias to be introduced is low. Finally, PSA data were only available for 2012; therefore, rates over 10 years are likely to be an underestimate because fewer dwellings would have been present in previous years. Nevertheless, again there is no evidence that these limitations in the dwelling counts were different between the buffers and is therefore unlikely to significantly bias the outcomes of the study.

\section{Conclusions}

This study highlights the importance for planning authorities to consider mosquito-borne disease risk when considering residential development applications. This is particularly important in WA where rapid population growth has resulted in acute pressure to develop land for residential purposes, with many new developments proposed in close proximity to mosquito breeding habitats due to the aesthetic value of living near water. However, as discussed previously, similar results have also been demonstrated for other diseases, so the potential health impact from mosquitoes on future residents should be considered wherever new developments are proposed across Australia and globally.

\section{Acknowledgment}

This study was funded by the Western Australia Department of Health.

\section{Author Disclosure Statement}

No competing financial interests exist.

\section{References}

Australian Bureau of Statistics. Regional Population Growth, Australia, 2012-13. Cat. no. 3218.0 Canberra: ABS; 2014. Available at www.abs.gov.au/ausstats/abs@.nsf/mf/3218.0/ Accessed April 10, 2014.

Department of the Environment. Peel-Yalgorup System. Last updated 24 January, 2011. Available at www.environment .gov.au/cgi-bin/wetlands/ramsardetails.pl?refcode $=36$ Accessed May 12, 2014.

Department of Planning. Planning for bushfire risk management. Last updated 29 August, 2014. Available at www.planning .wa.gov.au/publications/7183.asp Accessed October 8, 2014.

Erwin PC, Jones TF, Gerhardt RR, Halford SK, et al. La Crosse encephalitis in eastern Tennessee: Clinical, environmental, and entomological characteristics from a blinded cohort study. Am J Epidemiol 2002; 155:1060-1065.

Haque U, Huda M, Hossain A, Ahmed SM, et al. Spatial malaria epidemiology in Bangladeshi highlands. Malar J 2009; 8:185.

Hu W, Mengersen K, Dale P, Tong S. Difference in mosquito species (Diptera: Culicidae) and the transmission of Ross River virus between coastline and inland areas in Brisbane, Australia. Environ Entomol 2010; 39:88-97.

Jardine A, Neville PJ, Dent C, Webster C, et al. Ross River virus risk associated with dispersal of Aedes (Ochlerotatus) camptorhynchus (Thomson) from breeding habitat into surrounding residential areas: Muddy Lakes, Western Australia. Am J Trop Med Hyg 2014; 91:101-108.

Lindsay M, Oliveira N, Jasinska E, Johansen C, et al. An outbreak of Ross River virus disease in Southwestern Australia. Emerg Infect Dis 1996; 2:117-120.

Midega JT, Smith DL, Olotu A, Mwangangi JM, et al. Wind direction and proximity to larval sites determines malaria risk in Kilifi District in Kenya. Nat Commun 2012; 3:674.

Muhar A, Dale PE, Thalib L, Arito E. The spatial distribution of Ross River virus infections in Brisbane: Significance of residential location and relationships with vegetation types. Environ Health Prev Med 2000; 4:184-189.

Potter A, Johansen CA, Fenwick S, Reid SA, et al. The seroprevalence and factors associated with Ross River virus infection in western grey kangaroos (Macropus fuliginosus) in Western Australia. Vector Borne Zoonotic Dis 2014; 14 : 740-745.

Quantum GIS Development Team. Quantum GIS Geographic Information System. Open Source Geospatial Foundation Project, 2012.

Russell RC. Ross River virus: Ecology and distribution. Annu Rev Entomol 2002; 47:1-31.

Ryan PA, Alsemgeest D, Gatton ML, Kay BH. Ross River virus disease clusters and spatial relationship with mosquito biting exposure in Redland Shire, southern Queensland, Australia. J Med Entomol 2006; 43:1042-1059.

Staedke SG, Nottingham EW, Cox J, Kamya MR, et al. Short report: Proximity to mosquito breeding sites as a risk factor for clinical malaria episodes in an urban cohort of Ugandan children. Am J Trop Med Hyg 2003; 69:244-246.

Western Australian Planning Commission. Western Australia Tomorrow: Forecast Profile, Peel Planning Region. Population Report No. 7, 2006 to 2026; 2012. Available at 
www.planning.wa.gov.au/dop_pub_pdf/Peel(1).pdf Accessed April 10, 2014.

Wright AE. Report on the mosquito eradication campaign: survey of mosquitoes in the Manduarah region, Western Australia. Perth: Department of Health Western Australia, 1988.

Vally H, Peel M, Dowse GK, Cameron S, et al. Geographic Information Systems used to describe the link between the risk of Ross River virus infection and proximity to the Leschenault estuary, WA. Aust NZ J Public Health 2012; 36:229-235.
Address correspondence to: Andrew Jardine Mosquito-Borne Disease Control Environmental Health Hazards Unit Environmental Health Directorate Department of Health Western Australia Western Australia Australia

E-mail: Andrew.Jardine@health.wa.gov.au 